\title{
BMJ Open A patient cohort on long-term sequelae of sepsis survivors: study protocol of the Mid-German Sepsis Cohort
}

André Scherag, ${ }^{1,2}$ Christiane S Hartog, ${ }^{1,3}$ Carolin Fleischmann, ${ }^{1}$ Dominique Ouart,,${ }^{1,3}$ Franziska Hoffmann, ${ }^{1,3}$ Christian König, ${ }^{1,3}$ Miriam Kesselmeier, ${ }^{1,2}$ Sandra Fiedler, ${ }^{1,4}$ Monique Philipp, ${ }^{1,4}$ Anke Braune, ${ }^{1,4}$ Cornelia Eichhorn, ${ }^{1,4}$ Christin Gampe, ${ }^{1,4}$ Heike Romeike, ${ }^{1}$ Konrad Reinhart, ${ }^{1,3}$ for the Mid-German Sepsis Cohort investigators

To cite: Scherag A, Hartog CS, Fleischmann C, et al. A patient cohort on long-term sequelae of sepsis survivors: study protocol of the Mid-German Sepsis Cohort. BMJ Open 2017;7:e016827. doi:10.1136/ bmjopen-2017-016827

- Prepublication history for this paper is available online. To view these files please visit the journal online (http://dx.doi org/10.1136/bmjopen-2017016827).

Received 14 March 2017 Revised 4 May 2017 Accepted 12 June 2017

\section{(a) CrossMark}

${ }^{1}$ Integrated Research and Treatment Center, Center for Sepsis Control and Care (CSCC), Jena University Hospital, Jena, Thüringen, Germany

${ }^{2}$ Research Group Clinical Epidemiology, CSCC, Jena University Hospital, Jena, Thüringen, Germany

${ }^{3}$ Department for Anesthesiology and Intensive Care Medicine, Jena University Hospital, Jena, Thüringen, Germany

${ }^{4}$ Center for Clinical Studies, Jena University Hospital, Jena, Thüringen, Germany

\section{Correspondence to} Professor André Scherag; andre.scherag@med.uni-jena. de

\section{ABSTRACT}

Introduction An increasing number of patients survive sepsis; however, we lack valid data on the long-term impact on morbidity from prospective observational studies. Therefore, we designed an observational cohort to quantify mid-term and long-term functional disabilities after intensive care unit (ICU)-treated sepsis. Ultimately, findings for the Mid-German Sepsis Cohort (MSC) will serve as basis for the implementation of follow-up structures for patients with sepsis and help to increase quality of care for sepsis survivors.

Methods and analysis All patients surviving ICU-treated sepsis are eligible and are recruited from five study centres in Germany (acute care hospital setting in Jena, Halle/Saale, Leipzig, Bad Berka, Erfurt; large long-term acute care hospital and rehabilitation setting in Klinik Bavaria Kreischa). Screening is performed by trained study nurses. Data are collected on ICU management of sepsis. On written informed consent provided by patients or proxies, follow-up is carried out by trained research staff at 3, 6 and 12 months and yearly thereafter. The primary outcome is functional disability as assessed by (instrumental) activities of daily living. Other outcomes cover domains like mortality, cognitive, emotional and physical impairment, and resource use. The estimated sample size of 3000 ICU survivors is calculated to allow detection of relevant changes in the primary outcome in sepsis survivors longitudinally.

Ethics and dissemination The study is conducted according to the current version of the Declaration of Helsinki and has been approved by four local/federal responsible institutional ethics committees and by the respective federal data protection commissioners. Results of MSC will be fed back to the patients and published in peer-reviewed journals.

Trial registration number German Clinical Trials Registry DRKS00010050.

\section{INTRODUCTION}

Patients with sepsis experience life-threatening organ dysfunctions caused by a dysregulated host response to infection. ${ }^{1}$ Recent estimates of population incident rates from high-income
Strengths and limitations of this study

- More patients survive sepsis but data on mid-term and long-term consequences is scarce. The MidGerman Sepsis Cohort (MSC) is designed to close this gap by a rather comprehensive assessment of sepsis sequelae in a sample of $n=3000$ patients.

- Coverage of the majority of intensive-care-treated patients with sepsis with limited inclusion and exclusion criteria in Mid-Germany.

- MSC ascertainment is compatible with the new SEPSIS-3 definition.

- MSC is a non-interventional cohort, while some of the patients will require tailored postacute support.

countries ranged between 148 and 288 hospital-treated sepsis cases per 100000 person-years depending on disease severity; hospital mortality amounted to $26 \%$ for patients with (severe) sepsis during this period. ${ }^{2}$ Survivorship from sepsis is increasing, but the burden of survivorship is high: among 3years' (severe) sepsis survivors, up to $75 \%$ suffer from functional disability and about $17 \%$ suffer from cognitive impairment. ${ }^{3}$ Survivors also experience mortality rates, which are increased compared with propensity-score-matched cohorts of other hospitalised patients. ${ }^{4} \mathrm{~A}$ worldwide survey of sepsis survivors found that respondents suffered a range of impairment covering virtually every organ system and ability and deplored lack of services after acute care. ${ }^{5}$ There is a need for specific treatment strategies and rehabilitation concepts to deal with the long-term consequences of sepsis. ${ }^{6}$ However, such concepts are missing mainly because we lack prospectively collected and representative data on the nature, extent and impact of sepsis sequelae. Sepsis has therefore been called a 'hidden' healthcare disaster. ${ }^{7}$ 
A systematic review of long-term outcomes after sepsis included 30 studies up to February 2009. ${ }^{8}$ The studies identified focused on mortality and health-related quality of life (HRQOL) but did not cover a broader range of outcomes. Overall, the study quality of assessed studies was found to be low to moderate based on the US Preventive Task Force criteria. Specifically, it was impossible to determine to what degree the outcomes were attributable to sepsis rather than to comorbidities. The authors conclude that future observational and interventional studies should include longer follow-up periods and later endpoints to better understand the natural history of sepsis and the effect of interventions on patient morbidities. Despite new insights, ${ }^{49}$ these general remarks still apply. ${ }^{10}$

Consequently, we designed the Mid-German Sepsis Cohort (MSC) as a patient cohort to better and more completely quantify mid-term and long-term functional disabilities resulting from intensive care unit (ICU)treated sepsis. Ultimately, findings for MSC will serve as basis for the implementation of follow-up structures for sepsis patients and help to increase quality of care for sepsis survivors.

\section{METHODS AND ANALYSIS}

\section{Study design and setting}

MSC is designed as a patient multicentre cohort. Patients will be recruited from ICUs with minimal inclusion and no exclusion criteria to cover a wide scope of mid-term and long-term functional disabilities and consequences after ICU/hospital discharge.

After the initiation in March 2016, recruitment started aiming at including 3000 patients during a period of 3 years from ICUs in five study centres in Germany (acute care hospital setting in Jena, Halle/Saale, Leipzig, Bad Berka, Erfurt); in addition, we will recruit patients by a modified protocol in the setting of a large long-term acute care hospital and rehabilitation centre (Klinik Bavaria Kreischa). Patients are screened for eligibility on a daily basis by a team of trained ICU study nurses. Documentation using routine hospital data includes three assessments: $\mathrm{T}_{0}$ (onset of sepsis), $\mathrm{T}_{1}$ (ICU discharge) and $\mathrm{T}_{2}$ (hospital discharge). The follow-ups are planned after 3 $\left(\mathrm{FU}_{1}\right), 6\left(\mathrm{FU}_{2}\right)$ and 12 months $\left(\mathrm{FU}_{3}\right)$ and yearly thereafter up to 5 years (depending on additional funding) after ICU discharge. The follow-ups are conducted by trained study nurses, physicians or experienced medical students by phone call or face-to-face consultations by the study teams in Jena and Kreischa. Before the first follow-up at 3 months, survivors or their consenting proxies are sent an invitation letter; subsequently, a date is set up for a telephone interview. If the survivor is not able to participate in the interview because of cognitive impairment, the interview is performed with the legal proxy or main caregiver. If survivors or proxies cannot be contacted in time to participate at the first 3-month follow-up $\left(\mathrm{FU}_{1}\right)$, the subsequent interviews are nevertheless scheduled given that consent is not withdrawn. If possible, survivors are examined once over the study period in person.

\section{Eligibility criteria}

Patients aged $\geq 18$ years (at ICU discharge) with diagnosed (severe) sepsis and septic shock are enrolled. (Severe) sepsis is defined as clinically suspected or microbiologically proven infection and presence of at least one organ dysfunction due to infection. Septic shock is defined as persistent hypotension despite adequate volume resuscitation. Organ dysfunction and shock were diagnosed according to the current definitions of the German Sepsis Society, ${ }^{11}$ but documentation is such that the new SEPSIS- 3 criteria ${ }^{1}$ can also be applied.

Prior MSC enrolment is the only exclusion criterion in the ICU. Patients or proxies who are not fluent in German are excluded from the follow-up interviews.

\section{Acute hospital and follow-up assessments and outcomes}

Primary outcome is the change score $\left(\mathrm{T}_{1}\right.$ to $\left.\mathrm{FU}_{3}\right)$ of functional capacity assessed by activities of daily living (ADLs) and instrumental ADLs, which are valid instruments for the evaluation of the patients' self-care capacity. Secondary outcomes are related to changes or associations in the domains of HRQOL, cognitive dysfunction, post-traumatic stress symptoms, depression, fatigue, pain, insomnia, medication, use of medical services (rehabilitation, hospitalisation, out-patient therapy), recurrent infections and sepsis, reintegration and return to work, presumed cause of death and other long-term effects like dialysis, long-term ventilation and nursing care.

Table 1 is a summary of the documentation of acute care $\left(\mathrm{T}_{0}, \mathrm{~T}_{1}\right.$ and $\left.\mathrm{T}_{2}\right)$. It includes diagnostic and therapeutic measures, comorbidities, severity of illness scores ('Sequential Organ Failure Assessment' score), end-oflife decisions, length of ICU/hospital stay and time and cause of death if applicable.

A comprehensive list of physical, functional, emotional and mental sepsis outcomes documented at the follow-up appointments $\left(\mathrm{FU}_{1}, \mathrm{FU}_{2}, \mathrm{FU}_{3}\right.$, etc) was put together by the use of several sources (see table 2 for an overview). We performed a comprehensive literature search and used instruments of the German National Cohort. ${ }^{12}$ In addition, we drew on other Center for Sepsis Control and Care (CSCC) postacute experiences ${ }^{13} 14$ and refined the list by obtaining feedback from sepsis survivors and relatives from the German Sepsis Aid ${ }^{15}$ who advised on the comprehensiveness as well as relevance and also translated the final list of symptoms into lay terms (for instance, 'tingling' for paraesthesia, 'weakness of memory' or 'difficulty to concentrate' for cognitive impairments, etc). These lay terms are also used during the follow-up interview. We were also advised by Wes Ely (Vanderbilt University School of Medicine, Nashville, USA) on delirium monitoring and management in the ICU and assessment of pre-ICU cognitive functioning. All outcomes-except for hearing, tasting, smelling and swallowing-are measured by established and validated questionnaires. Depression 
Table 1 On-site ICU and hospital documentation of MSC

\section{Time of assessment} and domain

Documented items/content

\begin{tabular}{|c|c|}
\hline $\begin{array}{l}T_{0} \text { (on ICU, onset of } \\
\text { sepsis) }\end{array}$ & \\
\hline Demographic data & Age \\
\hline & Gender \\
\hline & Height \\
\hline & Weight \\
\hline & Insurance status \\
\hline Admission status & Date of hospital admission \\
\hline & Date of ICU admission \\
\hline & Admission type \\
\hline & Location prior to ICU admission \\
\hline & Prior ICU admission \\
\hline & $\begin{array}{l}\text { Current participation in an } \\
\text { intervention trial }\end{array}$ \\
\hline & Prior dialysis \\
\hline & Prior tracheostomy \\
\hline & Prior long-term ventilation \\
\hline & Prior dementia \\
\hline $\begin{array}{l}\text { (Severe) sepsis/ } \\
\text { septic shock criteria }\end{array}$ & $\begin{array}{l}\text { Presence of infection (community/ } \\
\text { hospital acquired, focus) }\end{array}$ \\
\hline & Presence of SIRS criteria \\
\hline & Presence of organ dysfunction \\
\hline & Septic shock \\
\hline & Date of sepsis onset \\
\hline Further information & Charlson Comorbidity Index \\
\hline & Glasgow Coma Scale \\
\hline
\end{tabular}

Selected vital signs (mean arterial blood pressure, $\mathrm{FiO}_{2}, \mathrm{PaO}_{2}$, urine output)

Administration of vasopressors (dose)

Selected lab results (creatinine, bilirubin, lactate, thrombocytes)

\begin{tabular}{cl}
\hline$T_{1}$ (ICU discharge) & \\
\hline Patient status & Time/cause of death \\
& Date of ICU discharge \\
& Presence of end-of-life care \\
& Presence/duration of delirium \\
& Presence/duration of ventilation \\
& $\begin{array}{l}\text { Presence/duration of replacement } \\
\text { therapy (lung, kidney, other organs) }\end{array}$ \\
& Vasopressor treatment \\
& Presence/duration of tracheostomy \\
& Presence/number of CPR \\
& $\begin{array}{l}\text { Presence/number of } \\
\text { physiotherapeutic treatment }\end{array}$ \\
\hline
\end{tabular}

Continued
Table 1 Continued

Time of assessment and domain

Documented items/content

Presence/number of erythrocyte concentrate treatment

Invasive focus reconstruction

Maximal Sequential Organ Failure Assessment score

Microbiology Existence of positive blood cultures Existence of relevant pathogens

\begin{tabular}{|c|c|}
\hline & $\begin{array}{l}\text { Existence of multi-resistant } \\
\text { pathogens }\end{array}$ \\
\hline \multirow[t]{2}{*}{ Further information } & Early Rehabilitation Index \\
\hline & Barthel Index \\
\hline \multicolumn{2}{|l|}{$T_{2}$ (hospital discharge) } \\
\hline \multirow[t]{8}{*}{ Patient status } & Time/cause of death \\
\hline & Date of hospital discharge \\
\hline & $\begin{array}{l}\text { Number of ICU episodes during } \\
\text { hospital stay }\end{array}$ \\
\hline & Discharge to \\
\hline & Weight at discharge \\
\hline & $\begin{array}{l}\text { Presence of tracheostomy at } \\
\text { discharge }\end{array}$ \\
\hline & Presence of ventilation at discharge \\
\hline & $\begin{array}{l}\text { Presence of kidney replacement } \\
\text { therapy at discharge }\end{array}$ \\
\hline
\end{tabular}

ICU, intensive care unit; MSC, Mid-German sepsis cohort; SIRS, systemic inflammatory response syndrome; CPR, cardiopulmonary resuscitation

and post-traumatic stress symptoms are measured by BSI- $18^{16}$ and PTSS-10. ${ }^{17}$ Cognitive function is assessed by a telephone version of the MoCA ${ }^{18-20}$ or $\operatorname{IQCODE}^{21}$ in proxy interviews. Fatigue and pain are measured by the Chalder Fatigue Scale ${ }^{22} 23$ and the Graded Chronic Pain Scale. ${ }^{24}$ EQ-5D-5L ${ }^{25}$ is used for assessment of the HRQOL prior to and after sepsis. Furthermore, survival status and other more specific domains of morbidity (eg, effects on tasting or sleeping) and healthcare utilisation as well as socio-economic effects and reintegration after sepsis are documented. Readmissions to hospitals, recurrent sepsis episodes, occurrence of infections, data on immunisation habits and persistence of multi-resistant infections are recorded. We aim for face-to-face contacts at least once during the study period to assess the Timed Up and Go test $^{26}$ and hand grip strength. ${ }^{27}$ We developed a written interview guide and refined the interview during a pilot test.

\section{Sample size calculation and data analysis plan}

We calculated the sample size necessary to detect relevant changes in the primary outcome in sepsis survivors longitudinally. In detail, we assumed a range of possible mean differences $\Delta$ in $\{0.5,0.6,0.7,0.8,0.9,1\}$ in a paired t-test 
Table 2 Central follow-up documentation and assessment tools of MSC

\section{Domain}

Sepsis perception

Current situation

\begin{tabular}{l} 
Medical information and socio-economic status \\
\hline ist of potential sepsis sequelae in patient-reported \\
terms
\end{tabular}

\section{Documented items/content}

"Do you know what sepsis is?"

"Do you know that you were treated for sepsis?"

Living conditions (location, change compared with prior to sepsis, care-giving needs)

Hospital readmissions

Recurrence of sepsis or severe infections

Use of rehabilitation or ambulant therapies

Medication

Presence of dialysis, tracheostomy, ileostomy, urinary catheter

Amputation of limbs due to sepsis

Vaccination status

Employment status (prior to sepsis, currently)

School education with graduation marks

Professional education

Family (marital status, spouse, children, siblings)

Rough estimate of (premorbid) general cognitive performance based on the study of Jahn et $a l^{29}$

Tobacco and alcohol consumption (prior to sepsis, currently)

Splenectomy/asplenia

Cognitive disabilities (attention deficit, disturbance of memory, speech disorder, impaired consciousness, lack of concentration)

Muscle and nerve weakness, stumbling, walking disability

Breathing problems

Weight loss

Depression, anxiety attacks, nightmares

Fatigue, listlessness

Personality changes

Sleep problems

Tingling in hands or feet

Multi-resistant pathogens

Need of care

Incontinence, dribbling

Mastery of daily life

Sexual problems

Ear, nose and throat symptoms (swallowing, smelling, hearing, tasting)

Other symptoms

Validated questionnaires related to specific domains

Quality of life (EQ-5D-5L) (prior to sepsis and currently)

$A D L$, (instrumental) ADL (prior to sepsis and currently)

Fatigue (Chalder Fatigue Scale)

Cognitive function (t-MoCA, IQCODE)

Post-traumatic stress disorder (PTSS 10)

Depression/anxiety/somatisation (BSI-18)

Pain (Graded Chronic Pain Scale according to Korff)

Sleep disorder (RIS)

In case of face-to-face consultation:

Physical mobility (Timed Up and Go test) ${ }^{26}$

Cognitive function (MoCA)

Hand grip strength ${ }^{27}$

Note that static items/content (like socio-economic status) is not again asked at subsequent follow-up assessments.

$A D L$, activities of daily living. 

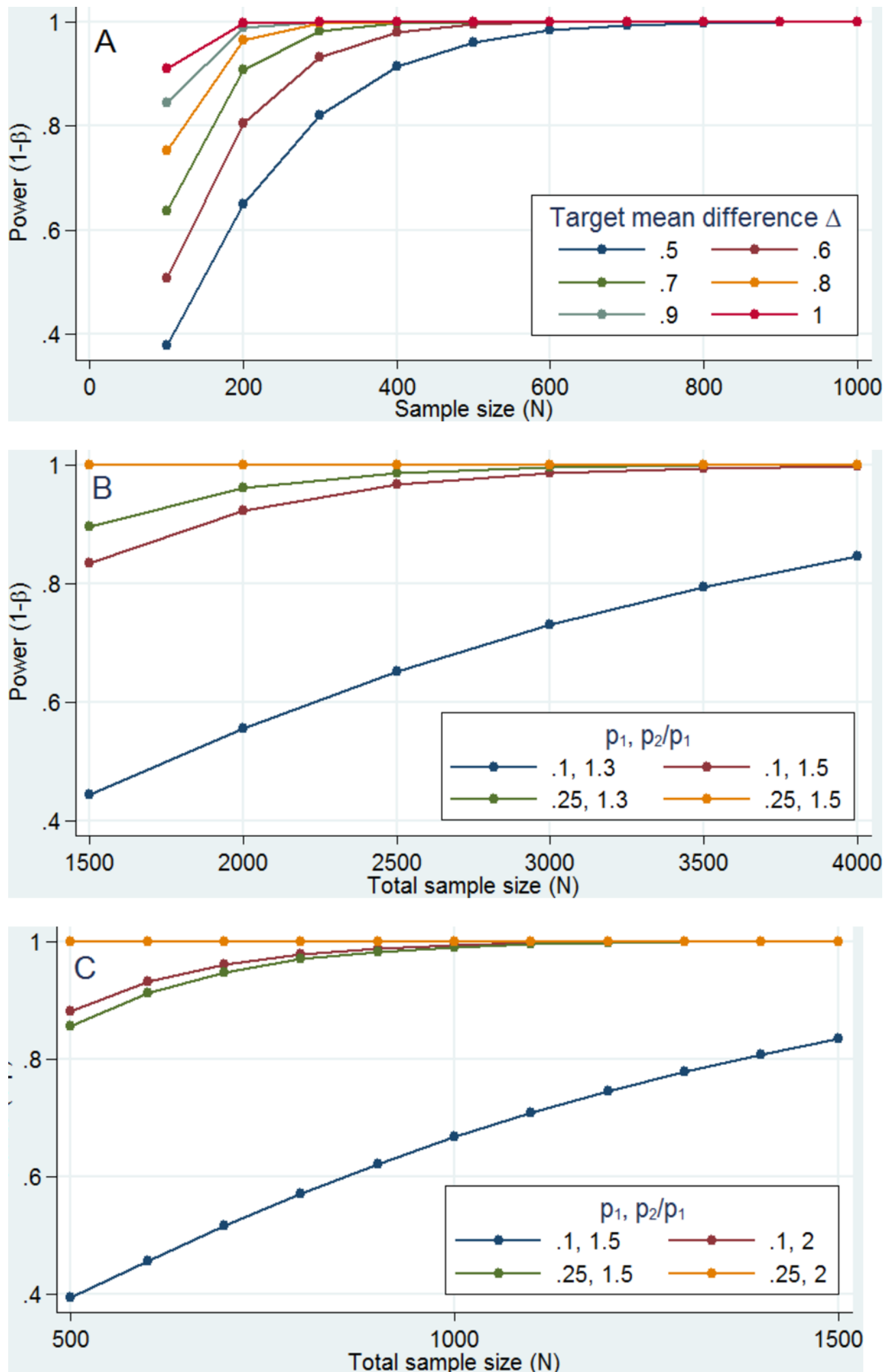

Figure 1 Power considerations for MSC. (A) Power for longitudinal changes against $\mathrm{N}$. (B) Power for associations against $\mathrm{N}$ at baseline $\left(\mathrm{T}_{0}, \mathrm{~T}_{1}\right)$. (C) Power for associations against $\mathrm{N}$ at 12-month follow-up $\left(\mathrm{FU}_{3}\right)$.

situation with a between-measure correlation of $\rho=0.5$ $\left(\mathrm{H}_{0}: \Delta=0\right.$ against $\mathrm{H}_{1}: \Delta \neq 0$ tested with $\alpha=0.05$; two sided) for a pretest mean of $8(\mathrm{SD}=3)$ for ADLs based on the data provided by Iwashyna et $a l .{ }^{28}$ We then varied the sample size that might be available for follow-ups from 100 to 1000 and plotted it against the comparison-wise power (figure 1A). In addition and more generally, we display a range of generic exposure-outcome associations (eg, prognostic factors to outcomes) for the initial ICU part of MSC (figure 1B) and expected proportion of survivors after 12 months (figure $1 \mathrm{C}$ ) for varying rates in exposed $\left(p_{2}\right)$ relative to unexposed $\left(p_{1}\right)$ patients (we assume a 1:1 
distribution of a binary exposure and applied a significance level of $\alpha=0.05$ (two sided) to a $\chi^{2}$-test) and realistic sample sizes. These considerations underline that about 3000 patients should be recruited to detect moderate and clinically relevant cross-sectional and longitudinal effects.

The main objective of MSC is to describe the course (distributions, prevalences) and potential prognostic factors of mortality, morbidity (including functional (dis) ability as well as physical, neuropsychological and neuromuscular disability) and postsepsis infection-related complications. Analyses of the main paper will therefore be explorative and more descriptive, applying state-ofthe-art methods (continuous variables, quartiles; count variables, absolute and relative frequencies; adequate regression models). All future analyses projects have to provide a precise research question and an analysis plan linked to it.

\section{ETHICS AND DISSEMINATION Informed consent}

No informed consent is required to collect routine documentation of acute care $\left(\mathrm{T}_{0}, \mathrm{~T}_{1}\right.$ and $\left.\mathrm{T}_{2}\right)$. Local study physicians obtain written informed consent for follow-up interviews from eligible patients (or their legal proxies) during the ICU or subsequent hospital stay. Patients or proxies are provided with written information about study aims and funding, study design and follow-up intervals, approximate duration of interviews, data protection and Institutional Review Board approval. Patients are assured that participation is voluntary and can be withdrawn at any time and that refusal to participate has no impact on medical treatment. If no legal proxy was established and written informed consent cannot be obtained during the acute hospital stay, family members receive a brochure with detailed information about MSC and are kindly asked to provide consent that the patient's contact information may be forwarded to the study centre so that the patient may be contacted later to obtain informed consent.

\section{Study registration, ethics review and data protection}

MSC is registered at the German Clinical Trials Register (DRKS00010050). It is conducted according to the current version of the Declaration of Helsinki and has been approved by four local/federal responsible institutional ethics committees and by the respective federal data protection commissioners. All data are collected with pseudonyms on electronic case report forms. Data capture takes place on the servers of Centre for Clinical Studies Jena at Jena University Hospital with 'OpenClinica', a validated study management software. OpenClinica meets all regulatory requirements (GCP, 21CFRPart11). By applying a hierarchical, role-based access control, an unauthorised access to patient data is impossible. Data are recorded in the study database via an encrypted data link (HTTPS) by use of data entry masks. It has an integrated audit trail that records any kind of data changes automatically. In order to ensure a pseudonymised analysis of data, each patient data set is given a unique patient identification number when being entered into the study data base. There is a backup of all data at regular intervals. During the study period, data quality is checked for errors electronically (eg, range checks) and on-site by experienced monitors.

\section{Access to data and dissemination}

Data access to the final cleaned data set is provided to all project applicants (primarily within the MSC investigator group) along with written use and access rules of the CSCC. To ensure confidentiality, data distributed to project applicants will be double pseudonymised and any directly identifying patient information will not be provided.

Results of this study will be presented at scientific conferences and published in peer-reviewed journals. Reporting will be in line with the STROBE statement. Authorship is granted according to the guidelines of the German Research Foundation. Furthermore, there will be patient meetings to disseminate study results.

\section{Perspective}

Survivorship after sepsis is an emerging problem. Sepsis outcomes are a complex interplay between patient demographics, co-morbidities, predisposition and risk factors, treatment in the ICU and sepsis-related organ dysfunction. Data from MSC may help to elucidate whether these are causal factors or confounders. While older age, gender and comorbidities have been described as risk factor for mortality after sepsis, it is unclear which risk factors drive the prolonged morbidity observed after sepsis. MSC will also provide data on readmissions and healthcare utilisation, thereby allowing estimation of the socio-economic dimension of sepsis sequelae. Lastly, the comprehensive and functional assessment of physical, mental and emotional symptoms will contribute to characterise the postsepsis syndrome and develop targeted interventions for rehabilitation and aftercare.

Collaborators Mid-German Sepsis Cohort investigators: The authors and Bauer M, Kortgen A (all Jena University Hospital), Schreiber T, Hüter L (both Zentralklinik Bad Berka GmbH), Oehmichen F (Klinik Bavaria Kreischa), Meier-Hellmann A (HELIOS Hospital Erfurt), Petros S, Bercker S, Stehr S (all University Hospital Leipzig), Bucher M, Nuding S, Frantz S (all University Hospital Halle (Saale)).

Contributors AS and KR contributed equally to this work. AS and KR obtained funding, supervised and guided the MSC. AS and KR, jointly with CSH, created the study concept and design. CSH supported the questionnaire selection. AS and MK were responsible for the statistical planning and the analyses. CSH, CF, DO, FH, CK and $\mathrm{HR}$ are responsible for conducting the central follow-up assessments including pilot tests of the interviews. SF supports the MSC as project manager. AB and CG are responsible for monitoring and educating the staff at the recruiting centres, while MP and CE are responsible for data management. AS, CF, CSH and KR wrote the manuscript; all other authors participated in its finalisation.

Funding MSC receives funding by the Integrated Research and Treatment Center, Center for Sepsis Control and Care (CSCC), at the Jena University Hospital and the Rudolf Presl GmbH \& Co, Kreischa. CSCC is funded by the German Ministry of Education and Research (BMBF no 01E01502). The funders have/had no role in 
study design, data collection and analysis, decision to publish or preparation of the future manuscripts.

Competing interests $\mathrm{CSH}$ receives a salary from the Rudolf Presl GmbH \& Co. Klinik Bavaria Rehabilitations KG as part-time director of patient- and familycentred care. KR is a paid advisor to Adrenomed AG, Henningsdorf b. Berlin, Germany, and holds equity at InflaRx GmbH, Jena, Germany.

Ethics approval Four local/federal responsible institutional ethics committees (ECs) including the EC of the Friedrich Schiller University (no 4669-01/16).

Provenance and peer review Not commissioned; externally peer reviewed.

Open Access This is an Open Access article distributed in accordance with the Creative Commons Attribution Non Commercial (CC BY-NC 4.0) license, which permits others to distribute, remix, adapt, build upon this work non-commercially, and license their derivative works on different terms, provided the original work is properly cited and the use is non-commercial. See: http://creativecommons.org/ licenses/by-nc/4.0/

(C) Article author(s) (or their employer(s) unless otherwise stated in the text of the article) 2017. All rights reserved. No commercial use is permitted unless otherwise expressly granted.

\section{REFERENCES}

1. Singer M, Deutschman CS, Seymour CW, et al. The Third International Consensus Definitions for Sepsis and Septic Shock (Sepsis-3). JAMA 2016;315:801-10.

2. Fleischmann $C$, Scherag A, Adhikari NK, et al. Assessment of global incidence and mortality of hospital-treated sepsis current estimates and limitations. Am J Respir Crit Care Med 2016;193:259-72.

3. Iwashyna TJ, Cooke CR, Wunsch H, et al. Population burden of long-term survivorship after severe sepsis in older Americans. J Am Geriatr Soc 2012;60:1070-7.

4. Prescott HC, Langa KM, Liu V, et al. Increased 1-year healthcare use in survivors of severe sepsis. Am J Respir Crit Care Med 2014;190:62-9.

5. Huang C, Daniels R, Lembo A, et al. Sepsis survivors' satisfaction with support services during and after their hospitalization. Crit Care Med 2016;44:425

6. RECOMMENDS to the Seventieth World Health Assembly. Secondary RECOMMENDS to the Seventieth World Health Assembly. http://bit.ly/whaebsepsisresolution.

7. Angus DC. The lingering consequences of sepsis: a hidden public health disaster? JAMA 2010;304:1833-4.

8. Winters BD, Eberlein M, Leung J, et al. Long-term mortality and quality of life in sepsis: a systematic review. Crit Care Med 2010;38:1276-83.

9. Yende S, Austin S, Rhodes A, et al. Long-term quality of life among survivors of severe sepsis: analyses of two international trials. Crit Care Med 2016;44:1461-6.

10. Iwashyna TJ, Angus DC. Declining case fatality rates for severe sepsis: good data bring good news with ambiguous implications. JAMA 2014;311:1295-7.

11. Reinhart K, Brunkhorst FM, Bone HG, et al. Prevention, diagnosis, therapy and follow-up care of sepsis: 1st revision of S-2k guidelines of the German Sepsis Society (Deutsche Sepsis-Gesellschaft e.V.
(DSG)) and the German Interdisciplinary Association of Intensive Care and Emergency Medicine (Deutsche Interdisziplinäre Vereinigung für Intensiv- und Notfallmedizin (DIVI)). Ger Med Sci 2010;8:Doc14.

12. German National Cohort (GNC) Consortium. The German National Cohort: aims, study design and organization. Eur J Epidemiol 2014;29:371-82.

13. Schmidt K, Worrack S, Von Korff M, et al. Effect of a primary care management intervention on mental health-related quality of life among survivors of sepsis: a randomized clinical trial. JAMA 2016;315:2703-11.

14. Mehlhorn J, Freytag A, Schmidt K, et al. Rehabilitation interventions for postintensive care syndrome: a systematic review. Crit Care Med 2014;42:1263-71.

15. The German Sepsis Aid. http://www.sepsis-hilfe.org/en/verein/ ueber-uns.html

16. Derogatis LR, Melisaratos $\mathrm{N}$. The brief symptom inventory: an introductory report. Psychol Med 1983;13:595-605.

17. Stoll C, Kapfhammer HP, Rothenhäusler HB, et al. Sensitivity and specificity of a screening test to document traumatic experiences and to diagnose post-traumatic stress disorder in ARDS patients after intensive care treatment. Intensive Care Med 1999;25:697-704.

18. Nasreddine ZS, Phillips NA, Bédirian V, et al. The Montreal Cognitive Assessment, MoCA: a brief screening tool for mild cognitive impairment. J Am Geriatr Soc 2005;53:695-9.

19. Pendlebury ST, Welch SJ, Cuthbertson FC, et al. Telephone assessment of cognition after transient ischemic attack and stroke: modified telephone interview of cognitive status and telephone Montreal Cognitive Assessment versus face-to-face Montreal Cognitive Assessment and neuropsychological battery. Stroke 2013;44:227-9.

20. Mackenrodt DWS, Eichstädt K, Rücker V, et al. Wie kurz darf eine kognitive testung sein? Validierung einer deutschen telefonischen Version Des Montreal Cognitive Assesments (MoCA). 89. Kongress der Deutschen Gesellschaft für Neurologie. 2016.

21. Ehrensperger MM, Berres M, Taylor KI, et al. Screening properties of the German IQCODE with a two-year time frame in $\mathrm{MCl}$ and early Alzheimer's disease. Int Psychogeriatr 2010;22:91-100.

22. Butler S, Chalder T, Ron M, et al. Cognitive behaviour therapy in chronic fatigue syndrome. J Neurol Neurosurg Psychiatry 1991;54:153-8.

23. Chalder T, Berelowitz G, Pawlikowska T, et al. Development of a fatigue scale. J Psychosom Res 1993;37:147-53.

24. Von Korff M, Ormel J, Keefe FJ, et al. Grading the severity of chronic pain. Pain 1992;50:133-49.

25. Herdman M, Gudex C, Lloyd A, et al. Development and preliminary testing of the new five-level version of EQ-5D (EQ-5D-5L). Qual Life Res 2011;20:1727-36.

26. Denehy L, Nordon-Craft A, Edbrooke L, et al. Outcome measures report different aspects of patient function three months following critical care. Intensive Care Med 2014;40:1862-9.

27. Ali NA, O'Brien JM, Hoffmann SP, et al. Midwest Critical Care Consortium. Acquired weakness, handgrip strength, and mortality in critically III patients. Am J Respir Crit Care Med 2008;178:261-8.

28. Iwashyna TJ, Ely EW, Smith DM, et al. Long-term cognitive impairment and functional disability among survivors of severe sepsis. JAMA 2010;304:1787-94.

29. Jahn T, Beitlich D, Hepp S, et al. Three socio-demographic equations for the estimation of the (premorbid) intelligence quotients according to Wechsler. Z Neuropsychol 2013;24:7-24. 